\title{
La co-docencia para el abordaje de la alfabetización académica: efectos en la forma- ción del profesorado disciplinar
}

\author{
Team teaching in an academic literacy approach: effects in the disciplinary lecturers' \\ training
}

Lucía Natale

e-mail: lnatale@campus.ungs.edu.ar

Universidad Nacional de General Sarmiento. Argentina

\section{Resumen}

En América Latina, las universidades han adoptado distintos dispositivos para promover la alfabetización académica. Son comunes los cursos para ingresantes y los centros de escritura. También se encuentra otra forma de enseñanza de la lectura, la escritura y la oralidad, situada en las materias de contenido, a cargo de un equipo formado por un docente disciplinar y un profesor de letras. En este artículo, se analizan los efectos de la adopción de este dispositivo de co-docencia en el marco del PRODEAC, un programa implementado en una universidad pública argentina. Para ello, se ha realizado una indagación cualitativa a partir de quince entrevistas en profundidad a profesores disciplinares que participaron del programa. El análisis, basado en la teoría empíricamente fundada, se concentra en los efectos del trabajo colaborativo reportados por dichos docentes. Se abordan dos aspectos: la enseñanza de la escritura en las asignaturas de contenido específico y las transformaciones en la planificación de los cursos. Los resultados señalan que el dispositivo de co-docencia contribuye a la reconsideración de la escritura y a la reorganización de los programas de las asignaturas. Los participantes manifiestan haber aprendido estrategias pedagógicas para incorporar la escritura como contenido y haber internalizado criterios para la elaboración de instrumentos y consignas de evaluación. Así, los resultados obtenidos permiten reconocer efectos positivos para la formación docente y la inclusión de la enseñanza de la escritura en las disciplinas. Asimismo, resultan relevantes para la formulación de programas institucionales de alfabetización académica.

Palabras clave: escritura académica; enseñanza; enseñanza en equipo; enseñanza superior.

\begin{abstract}
Latin-American universities have adopted different pedagogical strategies and devices to promote academic literacy. The most usual initiatives are first year writing courses and writing centers. Less common are collaborative teaching approaches in which a disciplinary specialist and an academic literacy lecturer work together to promote writing in the disciplines. This paper explores the effects of the implementation of this collaboration in raising the disciplinary lecturer's awareness about their tacit knowledge and the explicit teaching of academic literacy practices. Changes in their pedagogical planning are also examined. The study was conducted in an Argentinian public university, where an academic literacy institutional program based on team teaching is being developed since 2006. The paper draws on 15 interviews to disciplinary lecturers who participated in the program. The findings suggest that the collaborative teaching with an academic literacy lecturer enables disciplinary lecturers to have some critical distance from their disciplinary discourses and helps shift their planning and their teaching towards a more explicit pedagogy. This perspective appears to enable the explicit teaching of disciplinary literacies and could be useful in designing institutional projects.
\end{abstract}

Keywords: academic writing; teaching; team teaching; higher education.

Recibido / Received: 02-02-2020

Aceptado / Accepted: 26-05-2020

Publicado en línea / Published online: 01-07-2020

Cómo referenciar este artículo / How to reference this article:

Natale, L. (2020). La co-docencia para el abordaje de la alfabetización académica: efectos en la formación del profesorado disciplinar. Tendencias Pedagógicas, 36, pp. 104-116. doi: 10.15366/tp2020.36.08. 


\section{Introducción}

Desde finales del siglo XX, universidades de todo el mundo han comenzado a atender a los procesos de alfabetización académica (Carlino, 2013) del estudiantado, con el propósito de favorecer su inserción y permanencia en la educación superior. En este sentido, la mayor parte de las acciones han estado dirigidas a la mejora de sus procesos de comprensión y producción escrita. Por otro lado, se ha insistido en la necesidad de involucrar al colectivo docente para que incorpore la enseñanza de los géneros y discursos disciplinares como contenidos de las materias. Se busca así que el tratamiento de la lectura y la escritura no quede confinado a los primeros semestres, principalmente por el hecho de que se reconoce que esta instrucción inicial resulta insuficiente para comprender y producir textos que siguen convenciones específicas, vinculadas a los distintos campos del saber. Sin embargo, se reconoce que existen algunos obstáculos para que el profesorado disciplinar se haga cargo de esa tarea, sobre todo porque sus saberes sobre la escritura académica suelen ser tácitos, no conscientes, y porque no cuentan con herramientas específicas para encarar su enseñanza. Para resolver esta situación, se suelen organizar seminarios dirigidos al colectivo académico o proponer que trabajen de manera colaborativa, en parejas pedagógicas con especialistas en alfabetización académica.

En este artículo, se relevan algunos de los efectos que se generan a partir de la puesta en marcha de una enseñanza compartida entre el profesorado disciplinar y el especializado en lectura y escritura. Los datos que se presentan fueron obtenidos a través de entrevistas realizadas a seis profesores y nueve profesoras disciplinares que participaron de un programa institucional de una universidad pública argentina, que basa sus acciones en la co-docencia o enseñanza en equipo. El propósito del trabajo es dar cuenta de las transformaciones que reporta el profesorado disciplinar a partir de su participación en el programa. El artículo se concentra en dos aspectos: uno vinculado con aspectos involucrados en la enseñanza de la escritura y el otro, con la organización de la enseñanza y la evaluación de los contenidos específicos de la asignatura.

\section{La escritura de la escritura en la Universidad}

El interés por la enseñanza de la escritura en el nivel universitario puede ser rastreado a partir de 1870, a raíz de las transformaciones en el sistema universitario norteamericano. En el contexto de la creación de carreras profesionales, la escritura se transformó en una herramienta para la comunicación hacia el interior de audiencias especializadas, con géneros específicos (Russell, 2002,) y también en una cuestión de enseñanza a atender en el nivel superior. Sin embargo, las universidades no desarrollaron dispositivos para el tratamiento de la escritura en las disciplinas, sino que se encontró como solución la creación de cursos generales de composición ubicados en los primeros semestres. En estos, la escritura era tratada como una habilidad generalizable y elemental, independiente de los contenidos disciplinares, con foco en aspectos gramaticales y normativos. De esta manera, se dejó de lado «el status disciplinar de la escritura» (Russell, 2002, p. 7).

Un siglo después, estas primeras iniciativas se convirtieron en un campo de investigación y enseñanza, con la extensión de las propuestas de abordaje de la escritura en el nivel universitario, inicialmente en Estados Unidos y luego en países de todo el mundo (Thaiss, Bräuer, Carlino, GanobcsikWilliams, \& Sinha, 2012). En las universidades latinoamericanas en particular tienen lugar numerosas experiencias y se han desarrollado una cantidad importante de investigaciones, acompañadas por publicaciones especializadas y eventos científicos, junto con la creación de asociaciones y redes que reúnen académicos de distintos puntos del continente.

En este contexto, se han puesto en marcha distintos dispositivos para el abordaje de la escritura en la universidad. Se destacan los centros de escritura, en los que se ofrecen tutorías a estudiantes que demandan asistencia en la elaboración de un escrito; seminarios para la formación académica de profesorado en cuestiones vinculadas con la comprensión y producción de textos y los cursos basales dirigidos a las personas ingresantes, además de talleres de tesis en el contexto de los estudios de posgrado (Molina-Natera \& López-Gil, 2020; Núñez Cortés, 2013). En Argentina, el formato más extendido es el del curso inicial, ubicado en el primer año universitario o en talleres dirigidos a aspirantes a ingresar a la universidad. Estos cursos son comunes al conjunto de estudiantes de todas las carreras, sin que se distingan los géneros o discursos propios de los distintos campos del saber. En ellos se tratan cuestiones generales sobre la lectura y la escritura en la universidad, con foco en habilidades puntuales, ubicadas en el nivel textual (Carlino, 2013; Lea \& Street, 1998; Wingate, 2006). La 
búsqueda de información en la biblioteca y en internet, el uso de las normas de citación y de otras convenciones, la reformulación de fuentes para evitar el plagio, la organización de los textos en su nivel genérico y discursivo, con especial atención a la cohesión y la gramática son los contenidos comúnmente tratados. Tal como ha sido señalado (Carlino, 2013; Wingate, 2006), este tipo de instrucción no es suficiente, en tanto implican un primer nivel de abordaje, vinculado con el dominio de técnicas. En un plano de mayor profundidad, en el de la comprensión de la escritura académica, es preciso construir sentido en un discurso específico con el que los y las estudiantes no se han familiarizado aún, comprender cuáles de las fuentes son relevantes y por qué merecen ser citadas, entender de qué manera se presentan las evidencias para construir el conocimiento en un campo y, de ese modo, poder participar del discurso de los especialistas (Wingate, 2006). En este sentido, se entiende que las dificultades del estudiantado no se relacionan tanto con la falta de habilidades y técnicas que pueden ser rápidamente adquiridas, sino más bien con aspectos epistemológicos (Lea \& Street, 1998), con la comprensión de las lógicas disciplinares.

Para resolver el "divorcio" entre la enseñanza de la escritura y los contenidos en las asignaturas, se ha sugerido que los propios docentes de las materias se ocupen de enseñar a escribir de manera explícita (Duff, 2010; Monroe, 2006; Wingate, 2006, entre otros), puesto que son expertos en escribir los géneros de sus respectivos campos. Para ello, se recomienda poner en evidencia los presupuestos epistemológicos, los modos de construir el conocimiento, ubicar a los y las estudiantes en el rol de productores de conocimiento (antes que el de meros receptores) y mostrar los procedimientos retóricos para integrar la propia voz a los saberes ya establecidos (Wingate, 2006). Esto implica incluir la escritura como contenido de las asignaturas universitarias (Nesi \& Gardner, 2006), aunque se reconoce que esta tarea no resulta sencilla, ya que a menudo se piensa que el aprendizaje de la escritura se realiza en etapas previas a la universitaria. En lo que respecta a los propios académicos, se encuentra que su conocimiento sobre el discurso que se emplea en su campo disciplinar es a menudo tácito (Jacobs, 2005). Por otro lado, estos especialistas suelen plantear que ocuparse de la enseñanza de la escritura implicaría un trabajo adicional o que no están debidamente preparados para llevarlo adelante (Jacobs, 2005; Jenkins \& Wingate, 2015). Para poder resolver esta situación, se han sugerido dos estrategias. Una consiste en el desarrollo de cursos de sensibilización de los docentes de distintas carreras y la otra, el trabajo colaborativo entre los docentes de las asignaturas de contenido específico y especialistas en lectura y escritura de textos académicos (Wingate, 2015). De esta manera, se busca que el trabajo con la lectura y la escritura de los textos disciplinares se desarrolle de manera situada, con un abordaje específico, integrado a los contenidos de las asignaturas, y que esté distribuido a lo largo de todo el ciclo de formación, no ya «marginalizado» (Wingate, 2015) en los cursos iniciales.

El trabajo colaborativo o la conformación de parejas pedagógicas puede asumir distintas formas: el asesoramiento de los docentes lingüistas para la formulación de las consignas de trabajo, la elaboración de materiales pedagógicos y hojas de evaluación de los escritos, el dictado conjunto de las clases en las que se desarrolla una enseñanza explícita sobre la escritura de los géneros requeridos en la asignatura y, en ocasiones, la investigación compartida sobre las acciones desarrolladas (Coffin \& Donohue, 2014; Jacobs, 2005, 2010, 2015; Natale, 2013; Natale, Stagnaro, Pérez, \& Ríos, 2016; Purser, 2011; Purser, Skillen, Donohue, Deane, \& Peake, 2008; Skillen, 2006; Wingate, 2015; Wingate, Andon, \& Cogo, 2011)

Este dispositivo pedagógico ha sido evaluado como una herramienta que posibilitaría la transformación institucional en relación con el abordaje de la lectura y la escritura en las universidades (Wingate, 2018), en tanto implica el involucramiento de especialistas de distintas áreas en proyectos interdisciplinares y un sentimiento de pertenencia institucional (Jacobs, 2005), además de una organización interdisciplinaria y dialógica de las clases. En la literatura recolectada se encuentra, fundamentalmente, la descripción de las metodologías empleadas para esta «pedagogía colaborativa» (Jacobs, 2005). También se examinan los factores que influyen en la constitución de las parejas pedagógicas, como las formas de interacción, la naturaleza de las relaciones, las dinámicas internas de poder y la distribución de roles (Jacobs, 2010). Otro aspecto abordado en las investigaciones se refiere al modo en que las personas participantes (estudiantes y docentes) evalúan las experiencias llevadas adelante. En relación con esto, se ha encontrado que tanto especialistas disciplinares como estudiantes coinciden en su utilidad para mejorar el rendimiento académico del alumnado y la reducción de los índices de desgranamiento (Natale, Stagnaro, Pérez, \& Ríos, 2016; Wingate, Andon, \& Cogo, 2011). Asimismo, se han hallado mejoras en la calidad de los escritos producidos por los y las estudiantes (Wingate, Andon, \& Cogo, 2011. El alumnado ha señalado también que cuentan con más herramientas para organizar sus 
textos y adecuarse a normas, además de sentirse más seguros a la hora de encarar una producción escrita, lo que, según su perspectiva, también incide en sus trayectorias académicas y profesionales (Natale, Stagnaro, Pérez, \& Ríos, 2016). Por otro lado, el conjunto de docentes disciplinares reconocen que las interacciones y las negociaciones con los pares lingüistas les ha permitido explicitar conocimientos que permanecían como tácitos e implícitos en sus clases (Jacobs, 2005, 2015) y que la incorporación de algunos cambios en sus programaciones para atender a la escritura no les ha insumido mayor cantidad de tiempo en sus clases. Además, trabajar en favor de la alfabetización académica les permitió tener una mejor comprensión de las necesidades de sus estudiantes (Wingate, 2015) y poner en cuestión sus propias prácticas docentes (Natale, Stagnaro, Pérez, \& Ríos, 2016).

\section{El contexto del estudio}

El estudio que se presenta fue desarrollado en una universidad pública argentina, la Universidad Nacional de General Sarmiento (UNGS) en la que actualmente se dictan 22 carreras de grado: tres ingenierías, doce licenciaturas y siete profesorados, además de cuatro tecnicaturas.

Desde su creación, en 1992, se incluyeron en todos planes de carrera dos talleres obligatorios de lectura y escritura, en los que se abordan cuestiones generales sobre el discurso académico. El primero de ellos estaba ubicado en un curso de ingreso dirigido a aspirantes; desde 2019 se ha integrado a la curricula en el primer semestre. A continuación, se dicta un segundo taller en el que se profundizan las cuestiones generales y se abordan algunos géneros de formación como la respuesta de parcial y la monografía. Ambos talleres son dictados por docentes de Letras en espacios específicos. Por otro lado, desde 2005, comenzó a implementarse el Programa de Apoyo a la Lectura y la Escritura a lo largo del Curriculum (PRODEAC), que brindaba asistencia a los docentes de materias de distintas carreras que demandaban su intervención, sea a partir de la detección de dificultades de escritura en el estudiantado o porque los y las docentes deseaban introducir cambios en las actividades de lectura y escritura. Desde 2019, este programa se transformó en un espacio curricular denominado «Acompañamiento a la Lectura y la Escritura en las Disciplinas» (ALED), que obligatoriamente se inserta en dos asignaturas (intermedias o avanzadas) de las carreras para favorecer un trabajo continuo y transversal con la lectura y la escritura en las disciplinas.

Este dispositivo, iniciado por el PRODEAC y continuado en ALED, tiene como característica distintiva que las acciones de enseñanza de la lectura, la escritura y la oralidad tienen lugar en las mismas clases de las materias curriculares y no en espacios separados. Es decir, se trata de un abordaje situado en las disciplinas. Los contenidos sobre lectura y escritura se integran en los contenidos de las asignaturas: se parte de la idea de que las prácticas letradas y los géneros propios de una cultura disciplinar son inseparables de los conceptos, teorías y metodologías de cada campo de conocimiento. El dictado de estas clases se realiza a partir de la enseñanza en equipos o co-docencia. Esto significa que se encuentra a cargo de una pareja pedagógica conformada por docentes responsables de las asignaturas (especialistas en el campo en que se inscribe la materia) y un profesor o profesora del equipo PRODEAC/ALED (con título en Letras con amplia experiencia en el dictado de talleres de alfabetización académica). Ambas figuras, en forma conjunta, diseñan y proponen una secuencia de trabajo, un cronograma de intervención en las clases para desarrollar algunas de estas actividades: hacer lecturas compartidas de textos ejemplares para evidenciar los recursos lingüísticos que sirven para la realización de los géneros, guiar la planificación, redacción y revisión de textos, generar instancias de reflexión sobre la escritura en las disciplinas, proveer de retroalimentación en situaciones de exposición oral, por ejemplo.

En las clases, cada uno de los miembros de esta pareja pedagógica asume una forma de participación particular. Los y las docentes disciplinares, miembros activos de las distintas comunidades, aportan la perspectiva de los expertos en un campo de conocimiento y proveen información sobre los contextos en que circulan los textos y sobre los participantes y los escenarios en que se producen los intercambios. El o la docente de alfabetización académica, a su vez, parte de los conocimientos proporcionados por el docente disciplinar para elaborar una descripción lingüística del género discursivo que se requiere en la asignatura. Además de las clases propiamente dichas, existen otras instancias en las que se lleva adelante el trabajo colaborativo de estas parejas pedagógicas. A inicios de cada semestre, sus miembros se reúnen para seleccionar los géneros, describirlos a partir de ejemplares provistos por el o la docente disciplinar y acordar una secuencia de trabajo en las aulas. Luego, periódicamente, 
interactúan para realizar ajustes a la secuencia o evaluar conjuntamente las acciones implementadas. Por otro lado, tras las intervenciones en las clases y a lo largo de todo el semestre, los docentes de alfabetización académica realizan un seguimiento de los escritos del conjunto de estudiantes generalmente, a través del correo electrónico o de las aulas virtuales- y, de ser necesario, realizan tutorías individuales y/o grupales para andamiar el proceso de producción de los textos o para resolver dudas puntuales.

\section{Metodología}

Para este trabajo, se han realizado entrevistas en profundidad a seis profesores y nueve profesoras disciplinares de asignaturas de contenido específico que habían participado de las acciones desarrolladas en el marco del PRODEAC. Fueron recolectadas en los años 2017 y 2018, en instancias en las que se estaban evaluando las acciones del Programa en vistas a su incorporación formal en los planes de estudio de todas las carreras, lo que ocurrió en 2019. Para la selección de las y los entrevistados se buscó que hubiera docentes que dictan carreras de distintos campos del saber. Se realizaron preguntas vinculadas tanto con las motivaciones para incluir sus materias en el programa como con los efectos (positivos o negativos) que encontraban tras la implementación de las secuencias. Las entrevistas fueron administradas por la coordinadora del Programa, a quien los participantes conocían personalmente. Se realizaron tras la finalización de los semestres en que las asignaturas habían participado. Antes de iniciar la entrevista propiamente dicha, se explicitaron sus objetivos. Esto es: se buscaba conocer la perspectiva de los y las docentes disciplinares sobre el dispositivo y sus efectos en las asignaturas, con la aclaración de que no se pretendía evaluar la actuación del o la docente lingüista. Las conversaciones tuvieron lugar en un clima distendido, en las oficinas de los y las entrevistadas. En una instancia posterior, fueron transcriptas para identificar ejes conceptuales recurrentes, de acuerdo con la teoría empíricamente fundada (Glaser \& Strauss, 1967).

\section{Resultados}

A continuación, a partir del análisis de las entrevistas, exponemos los resultados organizados en dos ejes: los conocimientos que los y las docentes declaran haber desarrollado sobre la enseñanza de la escritura en las asignaturas y las transformaciones que dicen haber incorporado en sus planificaciones didácticas.

\subsection{La enseñanza de la escritura en las disciplinas}

Una primera cuestión que aparece en las entrevistas está relacionada con la enseñanza de la escritura en la universidad y con la distribución de los saberes y de los roles al interior del equipo. Como se ve en el extracto que sigue, una docente establece una diferenciación en cuanto a los conocimientos de cada uno de los miembros de la pareja:

«(1) Yo soy licenciada, soy Magister, pero no soy docente. Así que aprendí dando clases, pero no puedo enseñar lectoescritura. Yo puedo dar mucho apoyo en análisis de datos, pero no en escritura. No tengo herramientas o no tengo el tiempo para corregir holísticamente un informe. Yo no tengo esa capacidad. Ellas ven lo holístico» (Ec.- P 2).

En el ejemplo, la profesora hace referencia a su formación, y destaca que sus conocimientos formales, adquiridos en el grado y en el posgrado, se inscriben en un campo del saber y, por lo tanto, considera que tiene mucho que aportar a lo que hace a las metodologías para el análisis datos. En cuanto a los saberes pedagógicos, manifiesta que fueron desarrollados en la práctica, por lo que sugiere de alguna manera que no son sistemáticos. En relación con la escritura, si bien como especialista domina no solo contenidos específicos sino también los géneros de su campo, saberes que la habilitan para introducir a los estudiantes en el discurso disciplinar, manifiesta explícitamente que carece de herramientas para hacerlo. Les atribuye ese conocimiento a las docentes del PRODEAC («ellas»), quienes pueden realizar la tarea de una manera «holística». Así, en (1) se encuentra que la entrevistada establece una distinción entre la enseñanza de la producción escrita y la generación de conocimiento en su 
campo a partir del análisis de datos. Asimismo, marca la división de las tareas entre los miembros de la pareja pedagógica.

Esta división entre la elaboración de saberes y la escritura se refuerza en tanto caracteriza como «holístico» el proceso de revisar un escrito en todas sus dimensiones, mientras que su tarea se centraría en una parte; consistiría en observar la consistencia del análisis de los datos. La de los y las docentes del PRODEAC, por su parte, sería el de considerar el texto como una totalidad. Así, surge implícitamente una diferenciación entre saberes «holísticos» y otros más específicos, de carácter disciplinar.

Más allá de esto, la docente aclara que, independientemente de las herramientas, no tiene tiempo «para corregir holísticamente un informe». En esta breve frase se encuentra, por un lado, la idea de que la profesora entiende que el abordaje de la escritura insumiría un tiempo adicional de la clase. Asimismo, se observa que no define la tarea a realizar como «enseñanza», sino con la corrección. De esta manera, se insinúa que asocia la instrucción de la escritura con la mera evaluación; es decir, no contempla la posibilidad de proveer de lineamientos explícitos antes de que los y las estudiantes inicien la elaboración del informe.

Por otro lado, en la aclaración de que ella no es docente, la profesora deja entrever que la enseñanza de la escritura es asociada con un saber pedagógico, que estaría vinculado con la titulación. Esta observación se condice con los resultados de un estudio realizado por Jacobs (2010) en relación con los roles y responsabilidades de los miembros de la pareja pedagógica. La investigadora encontró que los y las docentes disciplinares asumían que el profesorado de escritura tenía un papel vinculado con el asesoramiento pedagógico, además de lo intrínsecamente relacionado con la alfabetización académica.

La diferenciación entre los conocimientos específicos de los miembros del equipo es planteada también en otros ejemplos, como el que sigue:

«(2) Hay un saber específico que traen los profes del PRODEAC que realizan un aporte fundamental a la cursada, que tiene que ver con la especificidad de los géneros académicos. En general los profes que damos la asignatura, que somos especialistas en otras cosas, sabemos esas cosas, pero de hacerlas, de hacer carrera académica. Antes de que existiera el PRODEAC, las reponíamos a partir de nuestra propia experiencia, pero sin tener ese saber específico. A mí personalmente me sirvió para ir incorporando estas especificidades a mis saberes» (Com. - A1).

En (2) se nota por un lado que existe la idea de que la experticia del profesorado de lectura y escritura se refiere a los géneros académicos, mientras que los y las disciplinares dominan «otras cosas» o, en todo caso, que su conocimiento de los textos de su campo es un saber tácito que han adquirido en sus prácticas como investigadores. Este saber, en su visión, puede ser puesto en juego, pero no explicitado sin alguna dificultad. Por otro lado, se encuentra que, según varios de los entrevistados, la participación en el programa y el trabajo conjunto con los especialistas en alfabetización académica es una vía para hacer más conscientes y sistemáticos los rasgos de los géneros y los discursos de su campo. Esta toma de conciencia hace que, paulatinamente los y las disciplinares los incorporen a su práctica docente. De este cambio da cuenta también el siguiente extracto:

«(3) Son cosas que uno las sabe, pero no las tiene conscientes para poder explicárselas a los alumnos. Yo les hago mucho hincapié en la lectura y les doy tips de escritura y de estructura de textos, pero ahora que pasé varias veces por el programa, las tengo más conscientes, y hasta hay cosas que incorporé yo, que antes no tenía. En mi bagaje como docente, te digo. Ahora explico todo esto incluso en posgrado. Aprendí y lo llevé al posgrado, porque hay cosas que parecen una pavada, pero tampoco lo tienen en el posgrado» (PS-A3).

En este testimonio nuevamente se constata el carácter tácito («uno las sabe, pero no las tiene conscientes para poder explicárselas a los estudiantes») que tiene el conocimiento sobre la producción escrita, como ha sido señalado reiteradamente. De acuerdo con Lillis (1997), las convenciones que gobiernan la escritura académica tienden a ser consideradas como «sentido común» (Lillis, 1997, p. 186), por lo que la enseñanza explícita de la organización de los textos científicos, sus normas y las discusiones que los atraviesan es poco frecuente en las clases universitarias, lo que genera algunos problemas. Según estudios etnográficos (Lillis, 1997), la falta de explicitación de las convenciones se convierte en una forma de exclusión para muchos estudiantes, especialmente para los que constituyen 
las "nuevas voces en la academia", aquellos que, provenientes de grupos sociales desaventajados, solo tuvieron la posibilidad de realizar estudios universitarios a partir de la ampliación del sistema, en décadas recientes.

Volviendo al ejemplo (3), a lo sumo, lo que se expone en las clases son «tips de escritura y de estructura de textos», lo que puede ser asimilado a lo que Wingate (2006) señala como meras técnicas, cuestiones de superficie de los textos académicos, sin que se logre una verdadera comprensión de las razones de su recurrencia.

Por otro lado, en el mismo fragmento, como en otros que se examinan más adelante, puede encontrarse un contrapunto entre un «antes» y un «ahora». En el testimonio del docente del ejemplo (3), estos adverbios marcan dos fases en relación con la enseñanza de la escritura. El «antes» señala un momento caracterizado por la falta de consciencia sobre los rasgos del lenguaje académico, mientras que el «ahora» hace referencia a la sistematización de esos conocimientos y a la posibilidad de explicarlos, luego de haber pasado varias veces por el programa. Puede decirse entonces que, a partir de su participación en el PRODEAC, este profesor ha podido poner en marcha procesos metalingüísticos y metadiscursivos que le han permitido verbalizar esos saberes y también traspolarlos a otras actividades docentes.

Finalmente, a partir de una de sus expresiones («cosas que parecen una pavada», una tontería) podría pensarse que, internamente, se ha producido un cambio en cuanto al status que este docente le asignaba a las convenciones de la escritura académica. De parecer «una pavada», lo que puede ser entendido como un aspecto superficial y conocido por los y las alumnas, ha sido reconsiderado como un contenido que debe ser enseñado.

En síntesis, según los ejemplos (2) y (3) se observó que los profesores disciplinares manifiestan haber desarrollado estrategias de enseñanza de la escritura relacionadas con la explicitación de aspectos que antes se presumían conocidos. Como se verá a continuación, en (4) y (5) se suma la incorporación de una nueva perspectiva sobre los estudiantes y sus posibilidades de aprendizaje. Ligado a ello, la docente expresa haber desarrollado otras modalidades de intervención para mejorar la producción de los estudiantes, más concretamente, las referidas a la retroalimentación de la escritura.

«(4) La profesora de PRODEAC trabajó mucho en clase, preparó varios materiales para Antropología Filosófica, con nuestra bibliografía, ha mostrado estilos, ha discutido esos estilos con los estudiantes y eso me ha servido mucho, porque nosotros antes corregíamos a partir de una serie de supuestos, que tenían que ver desde el recorte del tema, a veces nos parecían que faltaban determinados recursos, como bibliografía, que eran trabajos muy escolares, que a veces eran resúmenes. Y nosotros veíamos si tenía cita o no, las relaciones entre los acápites, pero no es que nos sentábamos con el equipo docente a ver qué tenía que tener un trabajo. Ahora con Gabriela fue distinto, porque estábamos todos con los estudiantes y discutíamos monografías de trabajos de otros años. Y los estudiantes hacían críticas, y yo, a la hora de corregir, antes lo veía, pero no me animaba, más allá de que había cosas que me hacían ruido, no me animaba a plantearlas como un problema de la monografía. Y después vi que los mismos estudiantes las veían y las planteaban y se veía que esas cosas iban en desmedro de la propia monografía. Yo antes lo veía y lo dejaba pasar...» (Fil-P1).

En el ejemplo puede reconocerse, por un lado, que la instrucción sobre la escritura que la profesora brindaba se limitaba a cuestiones casi de forma: la inclusión de citas y la relación entre los párrafos y apartados. Esto es, básicamente, se refería a la atención a reglas para la inclusión de voces y a la coherencia entre las partes que componen el escrito, por lo que no iban más allá del nivel textual. Según la misma entrevistada señala, en los comentarios que hacía a la hora de «corregir» (evaluar) los escritos, omitía señalar problemas más de fondo. Así, a la falta de explicitación y reflexión sobre las características de la producción académica se añade otra omisión, la de las sugerencias que podrían colaborar con el mejoramiento de los trabajos de los estudiantes. En este sentido, la posibilidad de que los y las estudiantes aprendan sobre la escritura disciplinar es retaceada en dos momentos de la instrucción: en la exposición de los aspectos que hacen a una buena producción académica y en la instancia de evaluación. La primera de estas omisiones podría deberse a la falta de conciencia sobre la dimensión genérica propia de toda producción. En cambio, la segunda parece estar asociada a representaciones de la docente sobre la capacidad de los estudiantes para comprenderlas o aceptarlas, en tanto señala que no se «animaba» a plantearlas. 
En el ejemplo (5), se encuentra que las intervenciones de la profesora de alfabetización académica permiten que la docente disciplinar reconozca saberes de los estudiantes.

\begin{abstract}
«(5) Era una monografía de grado... no sé... Yo creía que si el trabajo era muy bueno a nivel de contenido... yo por ahí esas cuestiones...las dejaba pasar. He calificado con 10, aunque era objetable. Y ahora vi que eso era criticable hasta para los alumnos. Y todo eso a mí me sirve, me sirve para corregir mejor un trabajo monográfico, porque nunca había visto ese trabajo tipo plenario con los estudiantes... y ahí pensé que hay cosas que si das orientaciones, podés calibrar, así que ahora puedo corregir mejor, porque ya veo que puedo decir que algo no está bien solo porque los contenidos estén. A mí me queda mucho más claro, puedo revisar un trabajo monográfico con mucha más seguridad, porque tuve una especialista que me hizo ver que por ahí hay cosas que sobran. Y yo antes por ahí pensaba... por ahí la estudiante no sabe... es una monografía de grado... Ahora hay formación e información. Y ahora ellos saben lo que estamos pidiendo. Yo me siento mucho más tranquila.» (Fil-P1).
\end{abstract}

De acuerdo con el ejemplo, entonces, observar la intervención de la docente de alfabetización académica hizo posible que la especialista disciplinar notara que los y las estudiantes contaban con herramientas para evaluar un texto y que, por ello, reconsiderara su potencial como lectores críticos y como escritores. Por otro lado, tuvo la oportunidad de asistir al despliegue de una estrategia de enseñanza de la escritura que define como «plenario». En esta situación, la disciplinar aparece posicionada en el rol de outsider (Jacobs, 2015), es decir como un sujeto ajeno a un campo de conocimiento y que, por tanto, no conoce de antemano aquello que está observando. Jacobs usa este término para caracterizar el papel de los especialistas en alfabetización académica en el trabajo colaborativo en parejas pedagógicas, quienes a través de la formulación de preguntas colaboran para que el docente disciplinar (el insider, en este caso) explicite las características del lenguaje que se usa en sus áreas de conocimiento, además de las reglas tácitas. Según Lillis y Rai (2011), los miembros de la pareja pedagógica alternan en el papel de insider y outsider y, sucesivamente, experimentan ambas condiciones.

Del ejemplo (5) surge en primer lugar que la actuación de la profesora de alfabetización académica funciona como un modelado de la enseñanza de la revisión de la escritura. En segundo lugar, se encuentra que la observación del plenario sirvió para que la disciplinar llegara a una conclusión de tipo pedagógico: la necesidad de plantear criterios de evaluación antes de que los y las estudiantes produzcan su texto, dejar en claro qué se les exigirá. Esta explicitación hace posible que, luego, la docente evalúe de manera más integral el trabajo, considerando tanto «los contenidos» como las dimensiones relativas a la escritura, en tanto habría brindado previamente «formación e información». A su vez, esta conclusión genera en ella una mayor confianza como docente y también hace que sea más exigente con las producciones.

El ejemplo (6) da cuenta de que la enseñanza colaborativa puede cooperar también con la comprensión de que la enseñanza de la escritura puede ser incorporada en las clases de las asignaturas sin que esto implique una actividad adicional.

«(6) Por ejemplo, cuando daban una clase sobre cómo hacer un PPT, yo pensé, "cuando nosotros hacemos un PPT podemos mostrarles cómo se hace una presentación oral, no solo darles el contenido, o cuando hacemos una clase sobre qué es la ciudad, les podemos decir: "yo las preguntas que me hago cuando leo, son estas". Podemos también hacerles ver qué preguntas nos hacemos, y de esa manera les estás enseñando. Por esto te decía, empecé a contarles el proceso que a lo mejor nosotros no nos lo explicitamos de esa manera. También empezamos a leer con ellos, pensando que era su primer texto académico, hacerles preguntas, qué nos quiere decir el texto, con quién está discutiendo... y bueno, cómo desarmar el texto, volver a hacerlo hasta que ellos lo vayan tomando como un ejercicio. Por eso te digo, es más integral, no es "no saben escribir, entonces llamo al PRODEAC ...". Entonces fueron experiencias más integrales.» (URB-A1).

En efecto, en (6) la docente de Urbanismo expresa que las intervenciones de su par de alfabetización académica le hicieron ver que ella misma podría orientar la producción. Resulta también interesante percibir que también vislumbra de qué manera puede brindar orientaciones: modelando ante los novatos las actividades que lleva adelante un experto. Como ejemplo, dice que puede dar cuenta de las preguntas que ella se hace mientras lee para poner al descubierto que un experto no realiza sus lecturas 
de modo automático, sino que se plantea interrogantes sobre los contenidos, sobre aspectos retóricos («qué nos quiere decir el texto») y sobre los debates del campo («con quién está discutiendo»). La docente define estas operaciones pedagógicas como «desarmar» la bibliografía y propone además que este tipo de prácticas constituyen una forma de instrucción. En palabras de la docente, se trata de empezar a «contarles el proceso». Así, la profesora se constituye como una suerte de modelo de lector y escritor académico.

Por último, en (6) también se encuentra una reconceptualización de las finalidades del programa y, a la vez, de cuáles son los objetivos de la enseñanza de la escritura en las disciplinas: no se trata de detectar y corregir errores de los y las estudiantes, como en un enfoque remedial, sino un abordaje «integral». Esto implica hacer visibles el entramado de un texto académico y también las operaciones que realiza un experto.

En síntesis, a partir del examen de distintos extractos de las entrevistas, encontramos que la observación de las estrategias didácticas de las y los docentes de alfabetización académica favorece que los y las disciplinares reconozcan distintos aspectos vinculados con la enseñanza de la escritura en las disciplinas. En primer lugar, les permite tomar consciencia de que no explicitaban conocimientos referidos a la escritura académica y que partían de una serie de supuestos, en parte porque los habían desarrollado en la práctica y, en parte, porque sentían que no tenían herramientas para enseñarlos. En segundo lugar, los y las disciplinares consignan que han comenzado a incorporar estrategias, como las vinculadas con la aclaración de los criterios de evaluación y con la inclusión de contenidos referidos a la dimensión discursiva en los momentos en que proveen retroalimentación y califican los trabajos. En tercer lugar, comienzan a delinear modos de sumar la instrucción sobre la lectura y la escritura de los textos disciplinares en las asignaturas de grado. Una de estas estrategias consiste en hacer visibles las que él o ella ponen en juego a la hora de leer y de producir un texto. Por último, se encuentran nuevas conceptualizaciones sobre lo que implica enseñar a escribir. Los y las disciplinares empiezan a entender que no se trata de mejorar aspectos superficiales, como en enfoques remediales, sino de una cuestión más integral u holística, según los términos usados por las y los entrevistados. Ligado a lo anterior, se reconsideran las finalidades del programa y se entiende que no se busca "corregir" los errores de los estudiantes, sino poner en evidencia las características propias de los géneros y discursos disciplinares. Se evidencia también que el programa buscaba colaborar con la enseñanza en las distintas fases que componen el proceso de producción escrita, además de cooperar con la formación de los y las docentes disciplinares para la incorporación de la escritura como contenido de las asignaturas.

\title{
5.2. En relación con la organización de la enseñanza de la asignatura
}

En el apartado anterior se han señalado algunas reconsideraciones sobre la enseñanza de la escritura en las disciplinas registradas en el discurso de los y las docentes entrevistados. En este, se reportan aquellas vinculadas con la reorganización de la planificación de los contenidos y de los instrumentos de evaluación. El ejemplo que sigue muestra algunos de estos cambios:

\begin{abstract}
«(7) Yo llegaba a la segunda mitad del semestre y me trababa, no podía avanzar. Es que los chicos no tienen las herramientas que yo necesito para trabajar. No podíamos interpretar los datos, tirar gráficos, hacer series de tiempo. Entonces Paula me re-ayudó en la planificación. Yo le expliqué y ella me preguntó: bueno, ¿qué son las cosas que los chicos tendrían que saber? ¿Qué cosas van a ver? Yo entonces ahí empecé a escribir: esto, esto y esto y ahí, conversando con ella, me di cuenta de que no lo había dado, pero lo esperaba. Entonces bueno, me ayudó a hacer la planificación mía y no tanto de los estudiantes. Cambié el cronograma. $\mathrm{Y}$ al final empecé a darle las herramientas y después a pedirle que hagan la lectura de los datos. A mí me destrabó esto.» (SIG-3).
\end{abstract}

En (7), la docente consigna que su planificación previa le generaba obstáculos para poder desarrollar la segunda parte de la materia y que esto se debía que había conocimientos que ella no había impartido, aunque «los esperaba». En este punto, se nota que los saberes que permanecen tácitos en las clases universitarias no se limitan a las convenciones de los discursos disciplinares, sino que la falta de explicitación puede también darse con contenidos que específicamente sirven para analizar datos, es decir para poder producir conocimiento nuevo. En este contexto, la profesora de alfabetización 
académica colaboró, mediante una serie de preguntas, en la reestructuración de los contenidos en la planificación de la materia. Las preguntas planteadas por la docente, que ocupaba aquí el rol de outsider, ayudaron a que la profesora disciplinar tomara conciencia de la necesidad de «cambiar el cronograma», darles «más herramientas» para poder demandar análisis de la información. En esta reconsideración el diálogo y la discusión aparecen como instrumentos útiles para promover la toma de conciencia de los docentes disciplinares sobre aspectos diversos.

Por otro lado, en el ejemplo se encuentra también que la disciplinar se reconoce a sí misma como destinataria del programa, en tanto señala «me ayudó a hacer la planificación mía y no tanto la de los estudiantes».

El interrogatorio y la negociación entre los miembros de la pareja pedagógica surge también como una herramienta para realizar ajustes en las consignas que se les solicitan a los estudiantes.

«(8) El profesor nos preguntaba qué esperábamos de los estudiantes, cuáles eran los objetivos por los cuales les pedíamos esa comparación, cuáles eran los mejores textos para hacer la comparación. Pensar, por ejemplo, nuevamente, la consigna. Por ahí nosotros la hacíamos muy general. Era: "Comparar estos textos en tales períodos históricos". Entonces, conversando, vimos dos cosas, que no les decíamos todo lo que íbamos a evaluar y que los estudiantes no tenían mucho de dónde agarrarse para hacer un buen trabajo, así que empezamos a dar más orientación.» (EP-P2).

En (8) se encuentra que los y las profesores disciplinares empiezan a tomar conciencia de que las consignas que formulaban no brindaban las suficientes informaciones como para que los y las estudiantes pudieran desarrollar sus escritos. Resultaban demasiado generales, en tanto no se explicitaban los requisitos de aprobación y tampoco se orientaba su producción, cuestiones que empiezan a ser visibles para el disciplinar a partir de las preguntas que le realizaba el o la docente de alfabetización académica cuando estaban planificando conjuntamente los instrumentos de evaluación. Las preguntas ponen al descubierto dimensiones que no se formulaban en la consigna y hacen que el disciplinar comience a vislumbrar los interrogantes que podrían planteárseles a los y las estudiantes. En este sentido, en el marco de la interacción y la negociación entre pares, se produce una suerte de desnaturalización de los enunciados que los y las disciplinares producen, ya que exigen considerar otros puntos de vista y las incógnitas que pueden suscitar en quienes reciben las consignas. Este aspecto no resulta menor, si se considera que estas son herramientas de mediación en los procesos de enseñanza y aprendizaje (Stagnaro, 2018) y que a menudo se producen discrepancias entre lo que el o la docente piensa que la consigna indica y lo que los y las estudiantes creen que se les solicita (Stagnaro y Chosco, 2013).

En otros ejemplos se encuentra también que los interrogantes de los y las docentes de alfabetización académica son un instrumento eficaz para que el cuerpo de docentes disciplinares comiencen a tomar en cuenta los propósitos pedagógicos y la audiencia a la que se dirigen.

«(9) Por ejemplo, yo les planteaba a los alumnos muchos objetivos, y ellas me decían: "No les des tantos objetivos. ¿Por qué preguntás esto? ¿Cuál es tu objetivo? Fijáte, es mucho. Después, cuando yo estaba armando un nuevo trabajo práctico, me aparecía en la cabeza la pregunta de Gabriela: ¿cuál es tu objetivo? ¿Por qué preguntás esto? Y por eso modifiqué mucho los trabajos prácticos y Gabriela me acompañó todo el tiempo en la revisión. "Fijáte si esto tiene sentido", me decía.» (SIG-1).

El ejemplo (9) permite ver que las preguntas del o la especialista en alfabetización académica son internalizadas por su par disciplinar y que sirven como guía cuando, en instancias posteriores al trabajo conjunto, se encuentran en la tarea de diseñar las consignas de las evaluaciones, como son los trabajos prácticos. En (9), el interrogante que recurrentemente aparece se refiere a los objetivos que se persiguen, lo que permite ajustar también las preguntas que el o la docente hace al grupo de estudiantes, a partir de la observación de su pertinencia para los propósitos específicos que se perseguían.

En el ejemplo (10), se visualizan otras dimensiones que comienzan a ser incorporadas por otro de los entrevistados: el nivel de avance de los estudiantes en sus carreras y, en función de eso, el grado de dificultad de las consignas y las ayudas que se requieren en cada nivel. 
«(10) Ahí vimos que era una materia muy inicial, que recién entraban, entonces nos hacían ver que a lo mejor les teníamos que dar un eje de comparación, como un puntapié inicial, y que ellos sumaran otros. En este sentido digo integral, no era solo cómo corregir o ver si escriben bien o mal, sino pensar en el marco, en pensar en todo un marco del trabajo. Eso lo hacía una participación integral.» (EP-A3).

En el ejemplo anterior resultan destacables dos aspectos. Por un lado, el docente señala que, en el diálogo con su par de alfabetización académica, toma conciencia de que, a la hora de elaborar las consignas de evaluación, es preciso considerar en qué instancia de sus estudios se encuentran los estudiantes. En este caso, considerando que se trata de una asignatura ubicada en los primeros estadios de la carrera, decidieron incorporar en la consigna un componente que oficiaba como ejemplo de una de las actividades que se estaba demandando. El ejemplo que se les brinda funciona entonces como una guía o andamiaje para que el estudiantado avance en la elaboración del escrito, que requiere que desarrollen nuevos conocimientos y habilidades.

Por otro lado, se encuentra que a partir de la intervención, el disciplinar comprende de mejor manera las funciones del programa («no era solo corregir o ver si escriben bien o mal»), sino más bien considerar el encuadre en que solicita un escrito, sus funciones, a quién se le está solicitando y, además, el papel que juegan el docente que lo demanda y las mediaciones que interpone para el logro de una buena producción.

Para sintetizar, en el análisis de los ejemplos de este apartado se ha encontrado que los disciplinares reportan nuevas tomas de conciencia, esta vez vinculadas con su oficio como docente. En efecto, la interacción en el interior de la pareja pedagógica, con otro que funciona como outsider, los docentes disciplinares dan cuenta de que han introducido cambios profundos en sus planificaciones, como la reestructuración de los contenidos; comienzan a vislumbrar que las consignas que elaboran pueden no contener toda la información que el alumnado necesita y, en función de eso, las ajustan y las enriquecen; reelaboran los instrumentos de evaluación de la materia y empiezan a contemplar como variable el grado de avance del grupo de estudiantes en sus carreras para calibrar el nivel de dificultad de las tareas que les proponen. En este sentido, puede decirse que la interacción con los y las docentes de alfabetización académica resulta también una vía para que los disciplinares desarrollen un grado mayor de conciencia sobre aspectos ligados a la práctica pedagógica en general y no únicamente en relación con el abordaje de la escritura en las disciplinas.

\section{Reflexiones finales}

Hasta aquí, a partir del análisis de fragmentos tomados de distintas entrevistas, se han podido reconocer algunos efectos positivos para la enseñanza en general y para la enseñanza de la escritura en las disciplinas en particular. Estos cambios se han producido en el marco del trabajo colaborativo, bajo la forma de co-docencia entre el profesorado disciplinar y otro especializado en lectura y escritura. Como ya señalamos, el dictado conjunto de las clases que se centraban en la enseñanza de la escritura han sido la ocasión para que los y las disciplinares comiencen a tomar conciencia de que los contenidos y las pautas para la producción escrita no eran explicitados en sus clases. Reconocieron que esos saberes provenían de su práctica y que no eran saberes conscientes y sistemáticos, por lo que no los exponían. En este punto, los resultados obtenidos son similares a los hallados por Jacobs (2005, 2007, 2015), en el sentido de que la interacción con el especialista en alfabetización académica llevó a este grupo de docentes a tomar distancia de su propio discurso y poder dar cuenta de los rasgos que lo caracterizan. Esta explicitación puede estar dada por el hecho de que se encontraban frente a colegas, que tenían la posibilidad de realizar preguntas sin que se ponga en juego su imagen. En cambio, como se ve en la práctica, los y las estudiantes no siempre sienten que están habilitados para solicitar aclaraciones, tal vez por temor a exponer su desconocimiento sobre un tema.

El cuerpo de disciplinares también ha planteado que carecen de herramientas específicas para la enseñanza de la escritura y que las pedagógicas más generales fueron desarrolladas en la práctica, con poca sistematicidad. En relación con este tema, dejaron en claro que la observación de las actividades que sus pares de alfabetización académica desarrollaban en el aula les había permitido conocer algunas estrategias y, en algunos casos, manifestaron que las habían utilizado con otros grupos de alumnos.

Por último, los y las disciplinares manifestaron que habían realizado cambios importantes en sus planificaciones y que comenzaron a reflexionar sobre sus consignas, tras comprender que debían 
brindar más detalles sobre la tarea. Asumieron que para la evaluación debían comunicar qué se esperaba de los trabajos y los criterios que seguirían. En relación con esto, puede decirse que la participación en el programa abarcó aspectos no considerados inicialmente y que tendría incidencia en el desarrollo de un conocimiento didáctico más general.

En un plano ya más subjetivo, las entrevistas permitieron observar que se produjeron algunas transformaciones sobre la valoración del alumnado y también en el desarrollo de una mayor autoconfianza en actividades docentes de suma relevancia, como la evaluación de los escritos estudiantiles.

De acuerdo con los testimonios relevados, las estrategias que han resultado más útiles para alcanzar estos resultados han sido, por un lado, el intercambio y la negociación con pares, como también encontró Jacobs (2005, 2007, 2015). Por otro lado, el modelado de las estrategias de enseñanza de la lectura y la escritura por parte del miembro del equipo especializado en alfabetización académica y la observación in situ realizada por su par disciplinar aparecen como estrategias eficaces para que los y las especialistas en contenido puedan comenzar a incorporar la enseñanza de la escritura en sus clases.

Para cerrar, más allá de que los testimonios recolectados son coincidentes, cabe consignar las limitaciones del estudio. En principio, se ha entrevistado a un conjunto acotado de docentes, en un número que podría extenderse, especialmente por la incorporación formal del espacio curricular a los planes de estudio. Por otro lado, es preciso recordar que todas las entrevistas fueron administradas en una misma universidad, por lo que podría pensarse que las expresiones pueden estar influidas por los discursos institucionales circulantes, que valoran explícitamente el rol de la escritura en la formación superior. Por otro lado, resultaría provechoso explorar la perspectiva de los y las especialistas en alfabetización académica. Esta indagación permitiría ampliar el panorama sobre los efectos de la constitución de equipos interdisciplinarios en la enseñanza de las prácticas de lectura, escritura y oralidad en las disciplinas.

\section{Referencias}

Carlino, P. (2013). Alfabetización académica diez años después. Revista Mexicana de Investigación Educativa, 18(57), pp. 355-381. Disponible en https://bit.ly/3ddTOi8.

Coffin, C., \& Donohue, J. (2014). A language as social semiotic based approach to teaching and learning in higher education. Chichester: Wiley.

Duff, P. (2010). Language socialization into academic discourse communities. Annual Review of Applied Linguistics, 30, pp. 169-192. doi: 10.1017/S0267190510000048

Glaser, B., \& Strauss, A. (1967). The discovery of grounded theory. Chicago: Aldine Press.

Jcobs, C. (2005). On being an insider on the outside: new spaces for integrating academic literacies. Teaching in Higher Education, 10(4), pp. 475-487. doi: 10.1080/13562510500239091

Jacobs, C. (2010). Collaboration as pedagogy: Consequences and implications for partnerships between communication and disciplinary specialists. Southern African Linguistics and Applied Language Studies, 28(3), pp. 227-237. doi: 10.2989/16073614.2010.545025.

Jacobs, C. (2015). Opening Up the Curriculum: Moving from The Normative to The Transformative in Teachers' Understandings of Disciplinary Literacy Practices. In Th. Lillis, K. Harrington, M. Lea, \& S. Mitchell (Eds.). Working with academic literacies: case studies towards transformative practice (pp. 131-142). Colorado: The WAC Clearinghouse/Parlor Press.

Jenkins, J., \& Wingate, U. (2015). Staff and students' perceptions of English language policies and practices in 'international universities: a case study from the UK. Higher Education Review, 47(2), pp. 47-73. Recuperado de https://bit.ly/2ZyhpX2

Lea, M., \& Street, B. (1998). Student writing in higher education: An Academic Literacies approach. Studies in Higher Education, 23(2), pp. 157-172. doi: 10.1080/03075079812331380364

Lillis, T. (1997). New voices in academia? The regulative nature of academic writing conventions. Language and Education, 11(3), pp. 182-199. doi: 10.1080/09500789708666727

Lillis, T., \& Rai, L. (2011). A case study of a research-based collaboration around writing in social work. Across the Disciplines, 8(3), pp. 1-10. Recuperado de https://bit.ly/2LWnogh

Molina-Natera, V., \& López, K. (2019). Estado de la cuestión de los centros y programas de escritura en Latinoamérica. Revista Colombiana de Educación, 78, pp. 97-120. doi: 10.17227/rce.num78-8066

Monroe, J. (Ed.) (2006). Local Knowledges, Local Practices: Writing in the Disciplines at Cornell. Pittsburgh: University of Pittsburgh Press. doi: 10.2307/j.ctt9qh4x1 
Natale, L. (2013). Integración de enfoques en un programa institucional para el desarrollo de la escritura académica y profesional. Revista Mexicana de Investigación Educativa, 18 (58), pp. 685-707. Recuperado de https://bit.ly/2WYfPfo

Natale, L., Stagnaro, D., Pérez, I., \& Ríos, L. (2016). El Programa de Desarrollo de Habilidades de Lectura y Escritura a lo largo de la Carrera de la Universidad Nacional de General Sarmiento, Argentina. En L. Natale, L., \& D. Stagnaro. Alfabetización académica. Un camino hacia la inclusión en el nivel superior (pp. 161-200). Los Polvorines: Ediciones UNGS.

Nesi, H., \& Gardner, S. (2006). Variation in disciplinary culture: University tutors' views on assessed writing tasks. British Studies in Applied Linguistics, 21, pp. 99-119. Recuperado de https://bit.ly/3eoltgR

Núñez Cortés, J. A. (2013). Una aproximación a los centros de escritura en Iberoamérica. Legenda, 17 (17), pp. 64-102. Recuperado de https://bit.ly/3eMh3Au

Purser, E. (2011). Developing academic literacy in context: trends in Australia. In M. Deane, \& P. O'Neill (Eds.), Writing in the disciplines (pp. 30-43). London: Palgrave Macmillan. doi: 10.1007/978$0-230-34451-8 \_3$

Purser, E., Skillen, J., Deane, M., Donohue, J., \& Peake, K. (2008). Developing academic literacy in context. Zeitschrift Schreiben, pp. 1-7. Recuperado de https://bit.ly/2zuORmv

Russell, D. R. (2002). Writing in the Academic Disciplines. 1870-1990: A Curricular History. Carbondale: Southern Illinois University Press.

Skillen, J. (2006) Teaching academic writing from the 'centre' in Australian universities. In L. Ganobcsik-Williams (Ed.), Teaching academic writing in UK higher education: Theories, practice and models (pp. 140 -153). London: Palgrave Macmillan. doi: 10.1007/978-0-230-20858-2_10

Stagnaro, D., \& Chosco Díaz, C. (2013). Discordancias entre expectativas docentes y representaciones de los estudiantes en torno a las tareas de escritura académica: diagnóstico y algunas propuestas de intervención. En L. Natale (Ed.) El semillero de la escritura. Las tareas escritas a lo largo de tres carreras de la UNGS (pp. 95-128). Los Polvorines: UNGS. Disponible para libre acceso y descarga en https://bit.ly/2LZMDOW

Stagnaro, D. (2018). Mediaciones docentes en la enseñanza de las disciplinas a través de la lectura y la escritura. En L. Natale, \& D. Stagnaro (Eds.). La lectura y la escritura en las disciplinas: lineamientos para su enseñanza (pp. 15-58). Los Polvorines: Ediciones UNGS. Recuperado de https://bit.ly/36vhRqu.

Thaiss, Ch., Bräuer, G., Carlino, P., Ganobcsik-Williams, L., \& Sinha, A. (Eds.) (2012). Writing programs worldwide: profiles of academic writing in many places. Anderson, SC: Parlor Press.

Wingate, U. (2006). Doing away with study skills. Teaching in Higher Education, 11(4), pp. 457-465. doi: $10.1080 / 13562510600874268$

Wingate, U. (2011). A comparison of 'additional' and 'embedded' approaches to teaching writing in the disciplines. In M. Deane, \& P. O’Neill (Eds.) Writing in the Disciplines (pp. 65-87). Basingstoke: Palgrave Macmillan.

Wingate, U. (2015). Academic literacy and student diversity. The case for inclusive practice. Bristol: Multilingual Matters.

Wingate, U. (2018). Academic literacy across the curriculum: Towards a collaborative instructional approach. Language Teacbing, 51(3), pp. 349-364. doi: 10.1017/S0261444816000264

Wingate, U., Andon, N., \& Cogo, A. (2011). Embedding academic writing instruction into subject teaching: a case study. Active Learning in Higher Education, 12(1), pp. 69-81. doi: $10.1177 / 1469787410387814$ 